\title{
Human Identification System Based on PCA Using Geometric Features of Teeth
}

\author{
Young-Suk Shin ${ }^{1}$ and Myung-Su Kim ${ }^{2}$ \\ ${ }^{1}$ Department of Information Communication Engineering, Chosun University, \\ \#375 Seosuk-dong, Dong-gu, Gwangju, 501-759, South Korea \\ ysshinachosun.ac. kr \\ 2 College of Dentistry, Chosun University, \#375 Seosuk-dong, Dong-gu, \\ Gwangju, 501-759, South Korea \\ msakimachosun.ac.kr
}

\begin{abstract}
We present a new human identification system based on PCA using geometric features of teeth such as the size and shape of the jaws, size of the teeth and teeth structure. In this paper we try to set forth the foundations of a biometric system for information encrypting of living people using dental features. To create a biometric matching system, a template based on principal component analysis(PCA) is created from dental data collected the plaster figures of teeth which were done at dental hospital, department of oral medicine. Templates of dental images based on PCA representation include the 100 principle components as the features for individual identification. The PCA basis vectors reflects well the features for individual identification in the whole of teeth and the part of teeth. The classification for human identification is generated based on the distance between the whole of teeth and the part of teeth with the nearest neighbor(NN) algorithm. The identification performance in 300 dental image is $97 \%$ for the part of teeth missed the right-molar and back teeth, $98.3 \%$ for the part of teeth missed the front teeth and $96.6 \%$ for the part of teeth missed the left-molar and back-teeth.
\end{abstract}

\section{Introduction}

Biometrics refers to the identity authentication of living people using their enduring physical or behavioural characteristics. Biometric identifiers are pieces of information encrypting a representation of a person's unique biological makeup. The most generalized biometric techniques include the recognition of fingerprints, faces, iris, retina, hand geometry, voice, signature and teeth $[1,2,3,4,5,6,7]$.

Jain and Chen[7] utilized dental radiographs for human identification. They studied a biometric system based on the distance between the antemortem and postmortem tooth shape. The dental radiographs have a number of challenges to overcome. For poor quality images where tooth contours are indiscernible, the shape extraction is a difficult problem for dental radiographs. Our algorithm utilizes information about differences in the size and shape of the jaws, size of the teeth and teeth structure.

In this paper, we present a new human identification system based on PCA using geometric features of teeth like the size and shape of the jaws, size of the teeth and 
teeth structure. First, we collected the plaster figures of teeth from the department of oral medicine in dental hospital. Second, we developed a representation of dental images based on PCA included the 100 principle components as the features for individual identification. Finally, the nearest neighbor algorithm for the classification of individual identification was applied.

\section{PCA Representation of Dental Features}

\subsection{Image Data}

Dental data was a database of the plaster figures of teeth which were done at Chosun University dental hospital, department of oral medicine. The data set contained images of 348 individuals of males and females. Each person has two images in a upper jaw and lower jaw. The data set used for research contained 300 gray level images in a upper jaw, each image using 800 by 600 pixels. Examples of the original images are shown in figure 1.
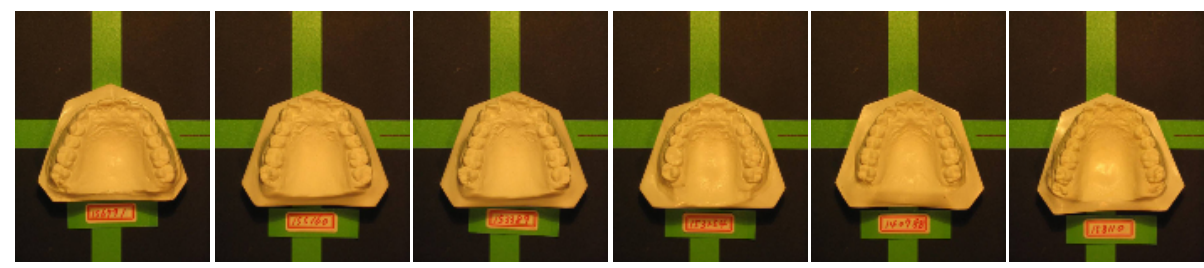

Fig. 1. Examples in a upper jaw from the dental database

\subsection{Preprocessing}

The dental images were centered with fixed coordinates locations, and then cropped and dug the palatine by semi-automatic method with a teeth template. Finally, The images were scaled to $30 \times 30$ pixels. Figure 2(b) shows the image dug the palatine with a teeth template(Figure 2(a)).

The luminance was normalized in two steps. First, a "sphering" step prior to principal component analysis is performed. The rows of the images were concatenated to produce $1 \times 900$ dimensional vectors. The row means are subtracted from the dataset, $X$. Then $X$ is passed through the zero-phase whitening filter, $V$, which is the inverse square root of the covariance matrix:

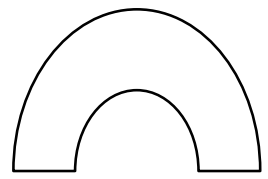

(a)

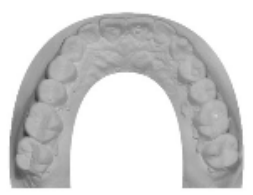

(b)

Fig. 2. (a) A teeth template (b) A dental image dug by a teeth template (a) 


$$
\begin{aligned}
& V=E\left\{X X^{T}\right\}^{-\frac{1}{2}} \\
& W=X V
\end{aligned}
$$

This indicates that the mean is set to zero and the variances are equalized as unit variances. Secondly, we subtract the local mean gray-scale value from the sphered each patch. From this process, $W$ removes much of the variability due to lightening.

\subsection{PCA Representation}

Some of the most successful algorithms applied PCA representation are "eigen faces[8]" and "holons[9]". These methods are based on learning mechanisms that are sensitive to the correlations in the images. PCA provides a dimensionality-reduced code that separates the correlations in the input. Atick and Redlich[10] have argued for such compact, decorrelated representations as a general coding strategy for the visual system. Redundancy reduction has been discussed in relation to the visual system at several levels. A first-order redundancy is mean luminance. The variance, a second order statistic, is the luminance contrast. PCA is a way of encoding second order dependencies in the input by rotating the axes to corresponding to directions of maximum covariance.

For individual identification based on dental feature, we employed the 100 PCA coefficients, $P_{n}$. The principal component representation of the set of images in $W$ in Equation(1) based on $P_{n}$ is defined as $Y_{n}=W * P_{n}$. The approximation of $W$ is obtained as:

$$
\bar{W}=Y_{n} * P_{n}^{T} .
$$

The columns of $\bar{W}$ contains the representational codes for the training images. The representational code for the test images was found by $\bar{W}_{\text {test }}=Y_{\text {test }} * P_{n}^{T}$ (see figure 3). Best performance for individual identification based on dental feature was obtained using 100 principal components.

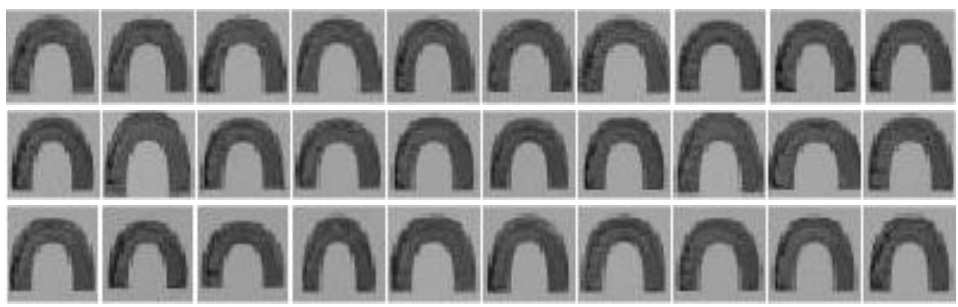

Fig. 3. PCA representation included the 100 principle components

\section{Results}

The first test verifies with 300 person image of upper jaw trained already. The recognition result was produced by 300 image trained previously showed $99.3 \%$ 
recognition rates. Since we had 300 person image of upper jaw in the training set, for testing, in total 900 image of 300 person excluded the three parts in the training set was used. The three parts consists of right-molar and back-teeth, left-molar and backteeth and front-teeth. Each individual has three different images and each part includes 300 person image. That is to say, the algorithms were tested for recognition under three different conditions: right-molar and back-teeth, left-molar and backteeth, and front-teeth.

Classification for individual identification was applied the nearest neighbor algorithm(NN). The principle of the $\mathrm{NN}$ algorithm is that of comparing input image patterns against a number of paradigms and then classifying the input pattern according to the class of the paradigm that gives the closest match.

The recognition performance was $97 \%$ for the part of teeth missed the right-molar and back-teeth, $98.3 \%$ for the part of teeth missed the front-teeth and $96.6 \%$ for the part of teeth missed the left-molar and back-teeth.

\section{Summary and Conclusions}

This paper propose a new biometric system for information encrypting of living people using dental features. By PCA representation based on geometric features of teeth such as the size and shape of the jaws, size of the teeth and teeth structure, our system can be used to authenticate an individual's identity by comparing a biometric reading from a person with a single stored template. This simulation demonstrates that PCA representation can solve a challenging problem such as a correct object recognition from the part shape in object recognition.

Our system extracts PCA representation included only 100 principle components from image scaled to 30x30 image and individual identification was successfully produced over $96 \%$ recognition rates. This results can reflect the fact that the holistic analysis is important for individual identification based on geometric features of teeth such as the size and shape of the jaws, size of the teeth and teeth structure. From the verification test with 300 person image of upper jaw trained already, we showed $99.3 \%$ recognition rates. It seems to be a erosion of detail shape of the jaws on the preprocessing caused by semi-automatic method with a teeth template. While we produced $100 \%$ recognition rates on the preprocessing by manual method in verification test trained already, the result on the preprocessing by semi-automatic method showed $99.3 \%$ recognition rates.

We suggest that in order to recognize the objects from the parts, the patterns within the scenes have to be holistic information reduced redundancy. In the future study we are planning to try individual identification on the preprocessing by automatic method.

\section{Acknowledgements}

This study was supported by research funds from Chosun University, 2005. 


\section{References}

1. Jain, A.K. Pankanti, S. Prabhakar, A., Ross, A.: Recent advances in fingerprint verification, Lecture Notes Comput. Sci. 2091 (2001) 182-190

2. Phillips, P.J.: Support vector machines applied to face recognition, Technical Report, NISTIR 6241, National Institute of Standards and Technology, 1999

3. Daugman, J., Downing, C.: Epigenetic randomness, complexity, and singularity of human iris patterns, Proc. Roy. Soc. 268 (2001) 1737-1740

4. Jain, A. K., Ross, A., Pankanti, S.: A prototype hand geometry-based verification system, Second International Conf. on Audio and Video-based Biometric Person Authentification, Washington DC, USA, March (1999)

5. Furui, S.: Recent advances in speaker recognition, in:J. Bigun, G.Borgeford (Eds.), Audio and Video-based Biometric Person Authentification, Springer, Berlin, (1997)

6. Nalwa, V.S.: Automatic on-line signature verification, Proc. IEEE 85 (2), (1997) 215-239

7. Jain, A. K., Chen, H.: Matching of dental X-ray images for human identification, Pattern Recognition 37(7), (2004) 1519-1532

8. Turk, M, Pentland, A. : Eigenfaces for recognition. Journal of Cognitive Neuroscience 3(1) (1991) 71-86

9. Cottrell, G., Metcalfe, J.: Face, gender and emotion recognition using holons. In Touretzky, D., editor, Advances in Neural information processing systems (3) San Maleo, CA. Morgan aufmann (1991) 564-571

10. Atic, J., Redlich, A.: What does the retina know about natural scenes?, Neural Computation (4) (1992) 196-210 